\title{
Buddhism and quantum physics: generality
}

\author{
Preecha P. Yupapin ${ }^{1,2, *}$ \\ ${ }^{1}$ Asian Quantum Life Institute, 92/395 Moo 11, Supakorn Village, Sai-noi Sub-district, Sai-noi District, Nonthaburi 11150, Thailand \\ *Corresponding author E-mail: kypreech@kmitl.ac.th \\ ${ }^{2}$ Interdisciplinary Research Center, Faculty of Science and Technology, Kasem Bundit University, Bangkok 10250, Thailand
}

Copyright $\odot 2015$ Preecha P. Yupapin. This is an open access article distributed under the Creative Commons Attribution License, which permits unrestricted use, distribution, and reproduction in any medium, provided the original work is properly cited.

\begin{abstract}
The generalized concept of Buddhism is presented in terms quantum physics, which can be used to practice and reach the noble human life, where the related Buddhism principles can be formulated by the quantum physics concept. This can lead to meet the well-known Buddhism practical states, where they are the contemplation and perception. Finally, the zero energy state (nirvana) can be achieved and realized, which is benefit to the un-limited brain performance condition and discussed.
\end{abstract}

Keywords: Quantum Buddhism; Brain Performance; Contemplation; Perception; Spirit Blueprint; Birth and Death Cycle.

\section{Introduction}

Generally, there are materials and non-materials (beings) existed in universe, where consequently, the basic elements of them are known as string and spirit for materials and beings (non-materials), respectively. The total energies of them are also conserved and formed by elements in the energy levels and sub-levels within the energy clusters. The non-material energy concentration is formed by the stars, while the being energy collection is formed by the Lord Buddha spirit [13]. Lord Buddha spirit and star energies are localized in Hilbert space. For the being life, the two zero states, birth and death cycle and spirit blueprint are observed within the Buddha energy clusters. From which, the energy is distinguished to be bright and dark energies that formed by the being bright and dark spirits, respectively. Principally, bright and dark energies are the entangled and orthogonal quantities. The collections of bright and dark energies are known as universe and black hole, respectively. Lord Buddha spirit (energy) is quantized and formed the energy cluster in being world, where alternatively, there is evil localized in the dark energy world (black hole). The spirit blueprint is constructed by the spirit pattern recognition, which means that the spirit blueprint is generated by the birth and death cycle activities [46], where they are turned to be the spirit blueprint after death, in which the rebirth of the suitable spirits with Five Aggregates and Four Elements can be formed and the resonant spirit signals can be performed.

\section{Principle}

Given $\psi_{\mathrm{i}}, \psi \mathrm{j}$ are the bright and dark spirit signals are formed by the Eigen functions in Hilbert space, where they are oscillated in time since the origin of big bang. The spirit energies of both quantities are presented in terms of Eigen value $E_{i}$ and $E_{j}$, respectively, from which they are generated by the spirit projection in Hilbert space and become the being the blueprints after death. The Eigen-value relationship can be expressed by

$\Delta \mathrm{E}_{\mathrm{i}} \Delta \mathrm{E}_{\mathrm{j}} \approx \mathrm{h}$

Where $\Delta \mathrm{E}_{\mathrm{i}}$ and $\Delta \mathrm{E}_{\mathrm{j}}$ are the bright and dark spirit energy exchanges, respectively, $\mathrm{h}$ is the Plank's constant. $\Delta \mathrm{E}$ is the real value. The ratio of $\Delta \mathrm{E}_{\mathrm{i}} / \Delta \mathrm{E}_{\mathrm{j}}$ value is defined as the signal to noise ratio of the bright and dark spirit signals, where when $\Delta \mathrm{E}_{\mathrm{j}}$ is approached zero, it means that there is no limitation of brain performance, where more detail is given later on. Given $\Delta \mathrm{E}_{\mathrm{i}}$ and $\Delta \mathrm{t}_{\mathrm{i}}$ are the bright spirit energy and oscillation time, respectively, then 
Where the state when $\Delta \mathrm{E}$ is approached zero, is the nirvana state, where the minimum energy is the simple harmonic motion with energy $\hbar \omega$, which is called the live nirvana, where the situation of perception is observed by several quantized energy states. The zero energy is the dead nirvana situation, from which the being body is turned to be relics. When $\Delta \mathrm{t}$ is approached zero, it is called the contemplation, which is the spirit stopping state in time that can be presented by several energy states, where they are the time independent situation (i.e. no time).

The bright and dark spirits are in the forms of orthogonal quantities that cannot be observed simultaneously, for instance, universe and black hole, Lord Buddha and Evil, happiness and suffering, where there are existed in the parity pair. The normalized quantities of the bright and dark spirit energies are formed by the spirit projection in the Hilbert space, which is given by the following relationship.

$<\Delta \psi \mathrm{i} \mid \Delta \psi \mathrm{j}>\leq \delta$

Where the maximum energy is occurred when $\delta=1$, alternatively when $\delta=0$. When $1<\delta<0$ presents the being activities within the birth and death cycle and the being blueprint after death, from which the next localized worlds will be chosen relatively to the former spirit pattern recognition, which is the quantized sates. The zero energy is equivalent to the nirvana, which is no re-birth.

From the Buddha history in the Tripitaka book [2], where there were three practical ways that Lord Buddha was gone through by practical ways before reaching the enlightenment, where they were (i) spirit oscillation time control by meditation, (ii) bright and dark spirit signal conversion by dietary, and (iii) the spirit energy control by Four Mindfulness Foundation. From my experiences, the brain performance could be increased and reached the state of unlimited brain performance, where the relationship between pure spirit and passion signals can be expressed by

Where $\mathrm{S}$ is the pure mind signals, $\mathrm{P}$ is the passion signals, when $\mathrm{P}$ is approached zero, from which the pure mind signal is approached infinity $(\infty)$, which means that the unlimited brain performance is achieved.

\section{Discussion}

To obtain the large pure bright spirit signals, the good practical way is the Four Mindfulness Foundation (FMF), which is incorporating with the practice of a Four Noble Truths, Three Characteristics, Four Routes of Successes (Accomplishments) and Eight Noble Paths. Finally, it is ended up by the clearly understanding of Five Aggregates and Perception, where the non-self-existence will lead to reach the enlightenment, which is become the noble people characteristics. Initially, people mind signals are formed by the bright and dark spirit signals spreading over the spirit spectrum, which is oscillated continuously with time in Hilbert space. Such spirit signals can be configured to be the non-dispersive soliton signals, which can be survived and localized in the Hilbert space. The increasing in bright spirit signals can be formed, in which the dark spirit signals can be suppressed within the spectrum by the given incorporating techniques, for instance, meditation and FMF by breath-in and out control method, where the concentration and mindfulness can be reached and achieved. Eventually, the single bright spirit peak can be obtained, from which the spirit energy can be controlled and reduced to approach zero by the last two techniques Five Aggregates Perception and Non-existence of the Three Characteristics, respectively. The spirit signal is vanished and energy remained in other form, which is changed to relics and satisfied the conservation of energy.

\section{Conclusion}

In this concept, the spirit is formed by the energy signal oscillates with time, which is called soul, where the stopping spirit with time (at rest) is called mind, while the last one is the spirit projection and consciousness. The spirit can be kept at rest by consideration of being dead, which is a method of Four Mindfulness Foundation, where the being spirit is afraid of being dead. The signal conversion between bright and dark spirit signals can be controlled by keeping the bright spirit signal always larger than the dark one, from which the dark spirit signals are suppressed under the bright spirit signal band, where alternatively, the dark spirit is turned up, from which the suffering is occurred. The noble people can be reached the state that the un-limited increasing in brain performance can be achieved.

\section{References}

[1] S.W. Hawking, "The Theory of Everything", 2007.

[2] E. William et al, Buddhist Philosophy: Essential Readings, Oxford University Press, 2009.

[3] P.P. Yupapin, Birth and Death Cycle: The Scientific Approach, Life Science Journal Research, 1(3) (2014) 76-79.

[4] S. Phunthawanunt and P.P. Yupapin, Science of Meditation and Therapy, Journal of Hamonized Researches in Applied Science, 2(3)(2014).

[5] P.P. Yupapin, Two Zero States in Buddhism: The Real Happiness, International Journal of Buddhism, 1(1) (2014) 1-3.

[6] P.P. Yupapin S. Pantian and and J. Ali, Čerenkov Radiation: The Space-Time Paradox, Nature \& Science, 11(12) (2013) 116-119. 